赵

Global Journals Inc.

(8)

GLOBAL JOURNAL OF RESEARCHES IN ENGINEERING: J

GENERAL ENGINEERING

Volume 19 Issue 1 Version 1.0 Year 2019

Type: Double Blind Peer Reviewed International Research Journal

Publisher: Global Journals

Online ISSN: 2249-4596 \& Print ISSN: 0975-5861

\title{
Remote Health Monitoring System using Wireless Body Area Network
}

\author{
By MD. Zahidul Islam, Sazzad Hossain Rafi \& MD. Murad Miah
}

University of Dhaka

Abstract- The rapid increase of wireless technologies and body area networks has enabled the continuous healthcare monitoring of patients from a remote location using various sensors. This paper describes a remote health monitoring system using WBAN where different sensors are used to collect a patient's vital signs. Once the data is received, the captured data are evaluated by the Arduino and sent to the patient's Android Smartphone using a Bluetooth module and a newly developed android application named Health Monitoring App. Data can be accessed by the patient himself, by doctor or patient's family members. In critical conditions such as when body temperature and pulse rate goes beyond a predefined value or patient fall down, then application from patient's smart phone triggers a message to deliver to the doctor or relatives with the location of the patient using built-in GPS in patient's phone. This remote health monitoring system is very feasible and cost-effective for a developing country like Bangladesh.

Keywords: body movement monitoring, health monitoring, wireless body area networks (WBAN), body condition analysis.

GJRE-J Classification: FOR Code:100510

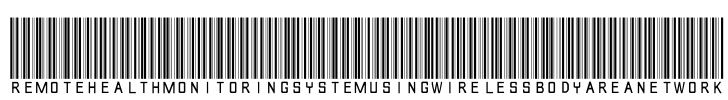

Strictly as per the compliance and regulations of:

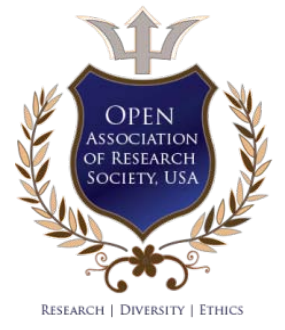

(C) 2019. MD. Zahidul Islam, Sazzad Hossain Rafi \& MD. Murad Miah. This is a research/review paper, distributed under the terms of the Creative Commons Attribution-Noncommercial 3.0 Unported License http://creativecommons.org/licenses/by-nc/3.0/), permitting all non commercial use, distribution, and reproduction in any medium, provided the original work is properly cited. 


\title{
Remote Health Monitoring System using Wireless Body Area Network
}

\author{
Md. Zahidul Islam ${ }^{\alpha}$, Sazzad Hossain Rafi ${ }^{\circ}$ \& Md. Murad Miah ${ }^{\rho}$
}

\begin{abstract}
The rapid increase of wireless technologies and body area networks has enabled the continuous healthcare monitoring of patients from a remote location using various sensors. This paper describes a remote health monitoring system using WBAN where different sensors are used to collect a patient's vital signs. Once the data is received, the captured data are evaluated by the Arduino and sent to the patient's Android Smartphone using a Bluetooth module and a newly developed android application named Health Monitoring App. Data can be accessed by the patient himself, by doctor or patient's family members. In critical conditions such as when body temperature and pulse rate goes beyond a predefined value or patient fall down, then application from patient's smart phone triggers a message to deliver to the doctor or relatives with the location of the patient using built-in GPS in patient's phone. This remote health monitoring system is very feasible and cost-effective for a developing country like Bangladesh.
\end{abstract}

Keywords: body movement monitoring, health monitoring, wireless body area networks (WBAN), body condition analysis.

\section{INTRODUCTION}

$\mathrm{B}$ ody area networks (BANs), also referred to as body sensor networks (BSNs), can be used for patient health monitoring. Wireless body area networks sense physiological activities of a human using a collection of sensor nodes which are smaller in size and have less power consumption. Then the data have to send to a microcontroller for further processing [1]. Body area networks benefit from the advancement of smart and inexpensive health monitoring systems [2]. They can also use as diagnostics, maintenance for specific health conditions and remote health observation. Body area networks employ features which are better suited for patient health monitoring compare with traditional wireless sensor networks (WSNs) [3]. Wireless body area networks are two types: in-body and on body. Both are frequently used for constant monitoring of the vital signs of a patient with proper diagnosis $[4,5]$.

\section{il. Remote Health Monitoring System}

In our developed system, a patient is monitor by the doctor or his family members from a remote place. The device will able to take data or vital signs such as body temperature, pulse rate, sense patient falling condition then send it to a microcontroller. Microcontroller further process the data, send to the patient's smart phone through a Bluetooth device. Where an android application used to check the data and this application will send a message to doctor or relative's phone after predefined time interval including information about body temperature, heart rate, body movement condition and patients location from Google map automatically. If heartbeat rate, body temperature goes higher than a predefined value or patient fall down then an automatic emergency message will go to doctor's or relative's phone from patient's mobile application using mobile networks.

There will be a couple of sensors like temperature sensor, pulse sensor, accelerometer and gyroscope sensor to detect the patient's temperature, heart rate and body movement information. For this system, sensors, Arduino Nano and a power source have used. An Arduino is use to evalute captured data and send to the patient's smartphone. The patient can move from one place to another with the device. Developed health monitoring system contains both a hardware part and a software part. The operation of our health monitoring system is shown below with a flow chart. 


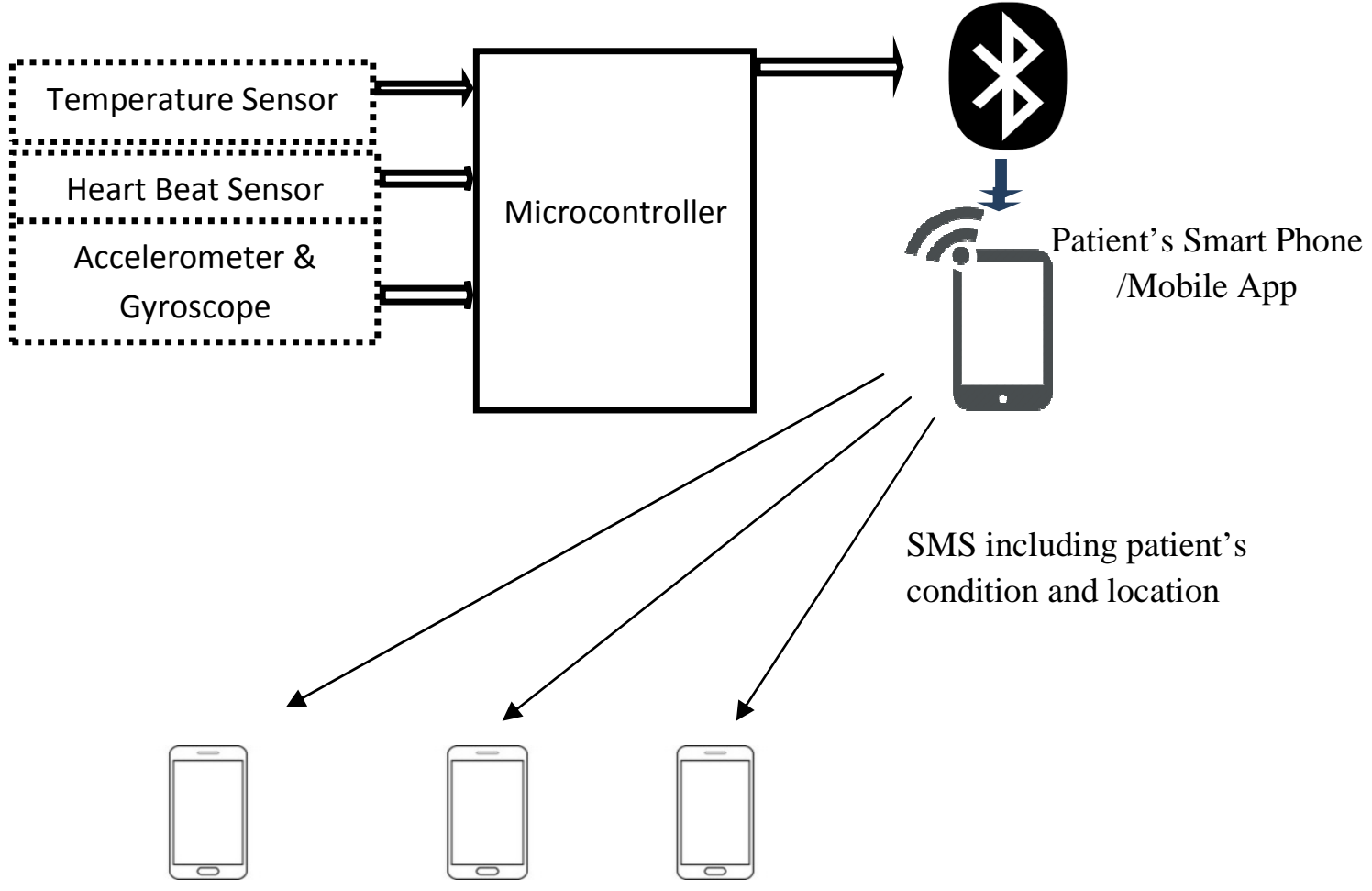

Relatives/Doctor's cell phone to monitor patient condition

Figure 1: Block diagram of health monitoring and Alerting System.

The sensors sense the data while the sensors are in patient's contact

Microcontroller receives the data through Bluetooth module and prepares to send

Patient's mobile application use to see body temperature, heart rate, body movement abnormalities and also send a sms to doctor or relative's phone after a certain time interval including above information and location

In critical condition, sms containing vital information and location are sent to doctor or relative's phone

Figure 2: Flow chart showing the operation of the remote health monitoring system.

ili. PARAmeters Estimation And Calculation

a) Body Temperature

LM35 temperature sensor is use to measure the temperature. LM35 is a precision IC temperature sensor with its output proportional to the temperature. Let $p$ is the value collected from Arduino analog pin and $q$ is a variable.

$$
\mathrm{q}=\frac{p * 5000}{1024}
$$

Celsius value $=\frac{q}{10}$

We can convert this Celsius value to Kelvin \& Fahrenheit value.

$$
\frac{C}{5}=\frac{F-32}{9}=\frac{K+273}{5}
$$


Where, $\mathrm{C}=$ Celsius value, $\mathrm{F}=$ Fahrenheit value, $\mathrm{K}=$ Kelvin value

As we get Celsius value, we can convert this to Fahrenheit value. As normally we use Fahrenheit to measure our body temperature. So it is necessary to convert from Celsius to Fahrenheit.

$$
\begin{aligned}
& \mathrm{F}=\frac{9 * C}{5}+32 \\
& \mathrm{~K}=\mathrm{C}+273
\end{aligned}
$$

By using those data, we can easily convert from one unit to another.

\section{b) Heart Beat Per Minute (BPM)}

The heartbeat sensor is based on the principle of photoplethysmography. It measures the change in volume of blood through any organ of the body which causes a change in the light intensity through that organ. The flow of blood volume is decided by the rate of heart pulses and since light is absorbed by blood, the signal pulses are equivalent to the heartbeat pulses. Pulse sensor SEN11574 is a very common sensor for measuring pulse rate. By just clipping the sensor to fingertip we can collect heart rate data.

\section{c) Body Movement}

If someone's body movement is abnomal then this device can detect the movement condition or fall. To detect normal or abnormal movement, MPU-6050 Accelerometer and Gyroscope has used in this system. The MPU6050 has an embedded 3-axis MEMS gyroscope, a 3-axis MEMS accelerometer. It is useful for some motion detection. For detecting how fast the acceleration is changing, we have used the concept of Standard deviation. Standard Deviation of the X-axis acceleration, Y-axis acceleration, Z-axis acceleration was measured by the function of a second. There arrive almost ten values in per second.

The formula for Standard deviation:

$$
\text { Standard deviation }=\sqrt{\sum(x-\bar{x})^{2} * \frac{1}{n}}
$$

Where, $\mathrm{x}=$ acceleration of a certain moment, $\bar{x}=$ average acceleration for a single second, $\mathrm{n}=$ number of acceleration data in a single second.

By using standard deviation, we can take some decision about the pattern of body movement. If the person is walking than the $\mathrm{x}, \mathrm{y}, \mathrm{z}$-axis acceleration is different from standing. By taking data in different condition and analyzing them, we can decide normal, abnormal body movement or falling situation.

\section{System Implementation}

\section{a) Hardware Implementation}

Wireless Body Area Network (WBAN) has been implemented here using Fritzing [6]. Various mathematical equations have been used to determine some parameters which are needed to analyze. Temperature sensor, Pulse sensor, Accelerometer and gyroscope sensor is connected the microcontroller. The microcontroller then sends the data to the patient's Smartphone. By using GPS, we can also detect the patient's location.

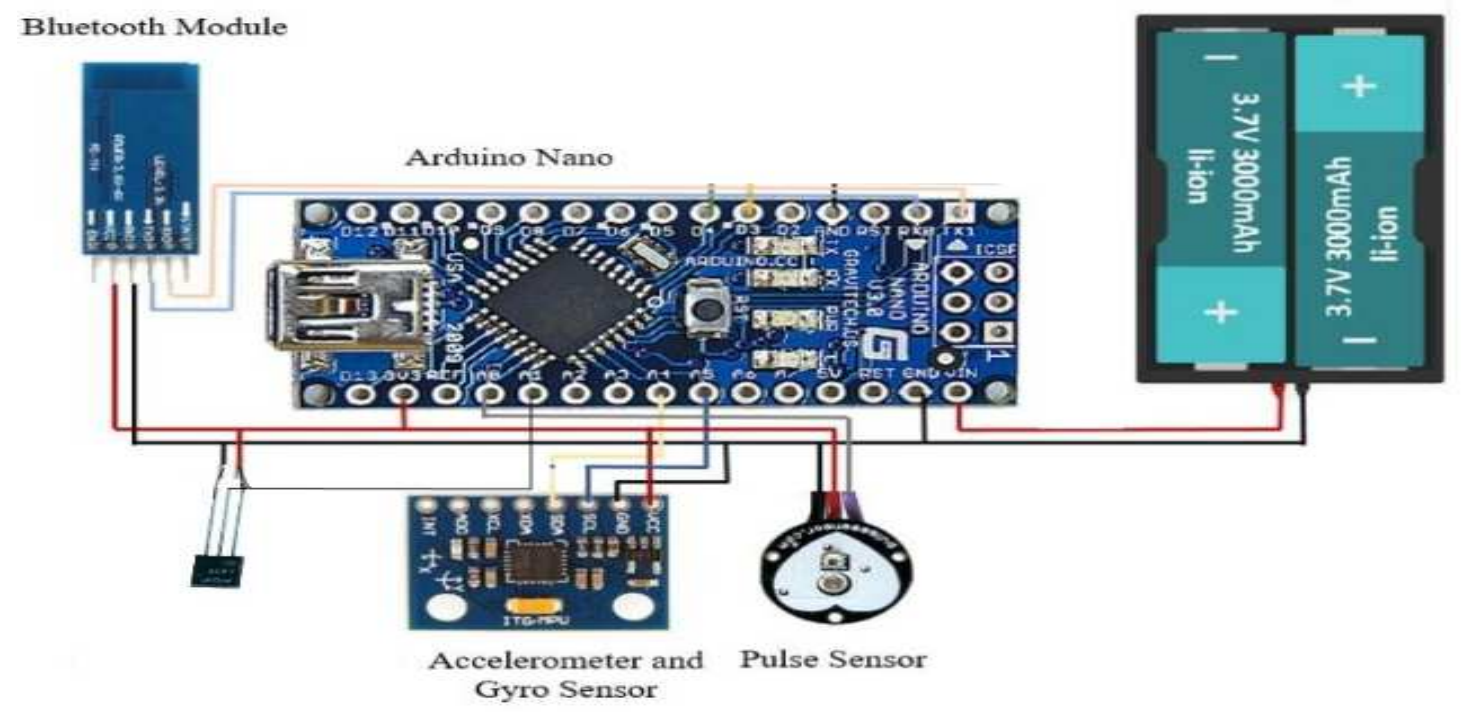

Figure 3: Implementation of hardware for remote patient monitoring system.

\section{b) Development of Mobile Application}

The primary task of our project was to take data from the sensors. The captured data are evaluated by the Arduino and sent to the patient's phone. A mobile application is needed to receive the data from a microcontroller and to send SMS to a doctor or relative's phone. Our fundamental objective was to give a stage to the patient, doctor, and family members to 
communicate simply without being available by physical. We have mainly focused on developing countries people where majority use Android device. Therefore it was another reason to choose Android mobile application. We have selected the name, 'Health Monitoring' for android the app. One app will belong to the patient only which will display his health parameters in real time as well as send vital information including patient's location via SMS service. Android Studio is used here to create an android application that will receive data from Arduino through Bluetooth device. Android Studio is the official Integrated Development Environment (IDE) for Android app development, based on IntelliJ IDEA. On top of IntelliJ's powerful code editor and developer tools, Android Studio offers even more features that enhance the productivity of the Android apps.

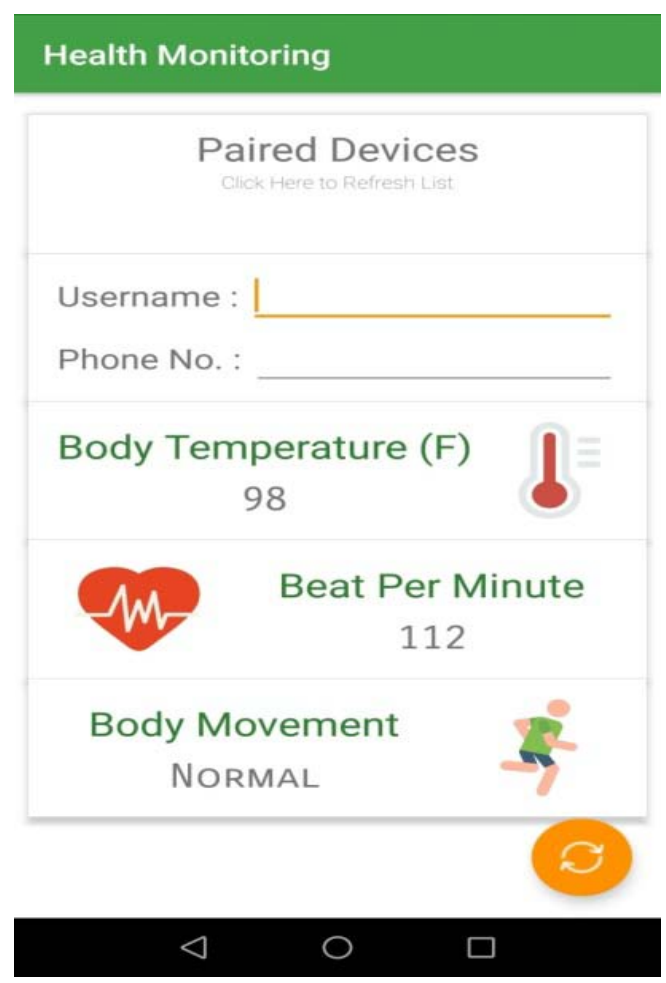

Figure 4: 'Health Monitoring' app to receive data from a microcontroller via Bluetooth module.

\section{Results and Analysis}

To verify whether our device is giving a better output or not we compared the outcome with the result that we get from a thermometer. As we are taking temperature and pulse rate, we need to make sure that our device produces an almost accurate output. We do the same process for falling condition analysis.

Table 1: Comparing the output with expected output (Body Temperature).

\begin{tabular}{|c|c|c|}
\hline Temperature using Thermometer & Temperature using Device & Percentage of Error \\
\hline $98 \mathrm{~F}$ & $99 \mathrm{~F}$ & $1.02 \%$ \\
\hline $100 \mathrm{~F}$ & $101 \mathrm{~F}$ & $1.00 \%$ \\
\hline $97 \mathrm{~F}$ & $98 \mathrm{~F}$ & $1.03 \%$ \\
\hline $98 \mathrm{~F}$ & $99 \mathrm{~F}$ & $1.02 \%$ \\
\hline
\end{tabular}

Table 2: Comparing the output with expected output (Pulse Rate).

\begin{tabular}{|c|c|c|}
\hline Pulse Rate (Manually) & Pulse Rate Using Device & Percentage of Error \\
\hline $60 \mathrm{bpm}$ & $65 \mathrm{bpm}$ & $8.33 \%$ \\
\hline $70 \mathrm{bpm}$ & $75 \mathrm{bpm}$ & $7.14 \%$ \\
\hline $75 \mathrm{bpm}$ & $78 \mathrm{bpm}$ & $4.00 \%$ \\
\hline $76 \mathrm{bpm}$ & $80 \mathrm{bpm}$ & $5.26 \%$ \\
\hline $110 \mathrm{bpm}$ & $112 \mathrm{bpm}$ & $1.82 \%$ \\
\hline
\end{tabular}


Table 3: Comparing the output with expected output (Fall Detection).

\begin{tabular}{|c|c|c|c|}
\hline $\begin{array}{c}\text { Number of trial for } \\
\text { fall detection }\end{array}$ & Number of Success & Number of Failure & Percent of Success \\
\hline 50 & 41 & 09 & $82 \%$ \\
\hline
\end{tabular}

In this developed system, there is an option of SMS that is send to the doctor's or family member's emergency phone number. It is an auto-generated message. An automatic message will send if the sensor

value exceeds a value. The conditions for triggering an SMS: (i) If Body Temperature is greater than 101 Degree Fahrenheit (ii) If Pulse Sensor Value is more than 120 or less than 60 and (iii) If Body Movement is abnormal.
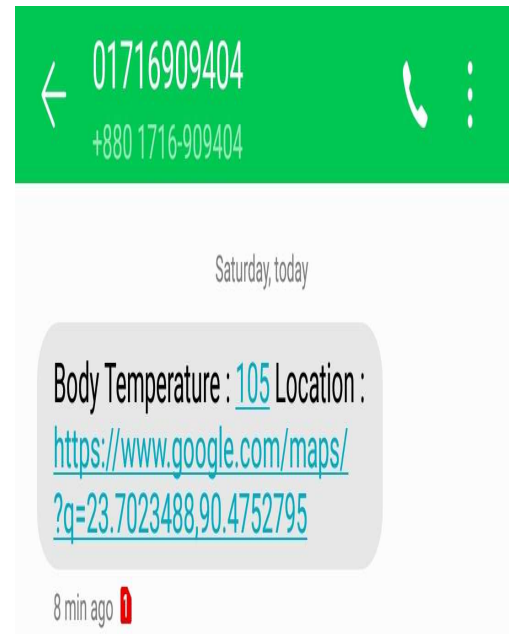

Figure: SMS for body temperature.

\section{Vi. Feasibility Analysis}

Feasibility analysis is used to aid the decision of whether or not to proceed with the proposed system. This system is independent and easy to use. Therefore it can be used at home or any remote location. In our country, the government and NGO's give a specific amount of money for healthcare development purpose. If patients use this device, that will be very cost effective and efficient for a developing country like ours.

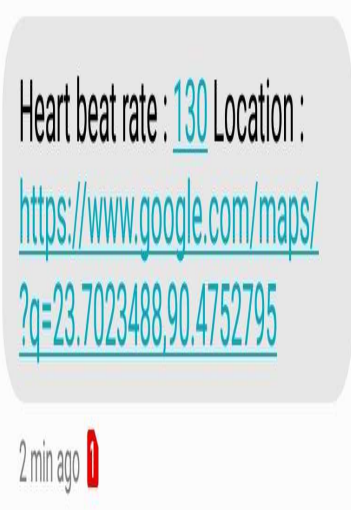

Figure: SMS for heart beat rate.

\section{Vil. Cost Analysis}

In developed countries like ours, there is few health monitoring devices which are very expensive. Our purpose is to make a device which is suitable for developing countries. See below for the total cost of this remote health monitoring system.

\begin{tabular}{|l|c|c|}
\hline \multicolumn{1}{|c|}{ Components } & Price (BDT) & Price (USD) \\
\hline Arduino Nano & 600.00 & 7.10 \\
\hline LM-35 temperature sensor & 60.00 & 0.72 \\
\hline SEN-00162pulse sensor & 1550.00 & 13.70 \\
\hline MPU-6050(3-Axis Accelerometer \& Gyroscope Sensor) & 180.00 & 2.15 \\
\hline HC-05 Bluetooth module & 280.00 & 3.34 \\
\hline Total Price & 2670.00 & 28.00 \\
\hline
\end{tabular}

\section{Viil. Conclusion}

Human body temperature, heart rate, body movement are the most important thing to analyze one's body condition. This paper illustrates and focuses on the sensors and their output result and remote patient monitoring. By this way, it is possible to know patient's body condition within a moment with exact location tracking system. People from anywhere and anytime can monitor patient especially elderly aged people. As there is not much complexity, it is easy to use. Because of being software-based, it has become very feasible.

\section{REFERENCES RÉFÉRENCES REFERENCIAS}

1. Milenkovic, A., Sanders, C., Otto, C., Sanders, C., Jovanov, E., "System architecture of a wireless body area sensor network for ubiquitous health monitoring". ACM Journal of Mobile Multimedia, 1(4): 307-326. 
2. Ullah, S., Higgins, H., Latre, B., Braem, B., Blondia, C., Moerman, I., Salem, S., Rahman, Z., Kwak, K. "A compressive survey of wireless body area networks. Journal of Medical Systems", 36(3): 1065-1094, 2012.

3. Gonzalez, S., Chen, MVasilakos, A., Cao, H., Leung, V. Body area networks: A survey. Mobile Networks and Applications, 16(2): 171-193, 2011.

4. Khan, P., Ullah, S., Ullah, N., Salem, S., Higgins, H., Kwak, K. (2009) A review of wireless body area networks for medical applications. Journal of Communications, Network and System Sciences, 2: 797-803.

5. E. H. Ashraf Darwish, "Wearable and Implantable Wireless Sensor Network Solutions for Healthcare Monitoring," Sensors, 2011.

$6 \quad$ 6. https://en.wikipedia.org/wiki/Fritzing (Last accessed on December 02, 2018). 